\title{
Macroscopic Findings Original Result
}

National Cancer Institute

\section{Source}

National Cancer Institute. Macroscopic Findings Original Result. NCI Thesaurus. Code

C119863.

The outcome of the macroscopic findings assessment as originally received or collected. 